\title{
WATER QUALITY IN CUTAWAY PEATLAND LAKES IN SEDA MIRE, LATVIA
}

\author{
Māris Kḷaviṇš*, Ilga Kokorīe*, Gunta Sprinğ́e*, Agnija Skuja**, Elga Parele***, \\ Valērijs Rodinovs ${ }^{\star \star \star}$, Ivars Druvietis ${ }^{* \star}$, Solvita Strāḳe ${ }^{* \star \star *}$, and Andris Urtāns ${ }^{\star \star \star * \star}$ \\ * Faculty of Geography and Earth Sciences, University of Latvia, Raina bulv. 19, LV-1586, Rīga, LATVIA; \\ e-mail: maris.klavins@lu.lv \\ ${ }^{* *}$ Faculty of Biology, University of Latvia, Kronvalda bulv. 4, LV-1586, Rìga, LATVIA \\ *** Institute of Biology of University of Latvia, 3 Miera iela, LV-2169, Salaspils, LATVIA \\ **** Latvian Institute of Aquatic Ecology, Daugavgrivas iela 8, LV-1048, Rīga, LATVIA \\ **** North Vidzeme Biosphere Reserve, Rīgas iela 10a, LV-4033, Salacgrīva, LATVIA
}

Contributed by Māris Kḷaviṇš

\begin{abstract}
Transformation into shallow lakes can be a major post-harvesting land-use option for cutaway peatlands. The aim of our study was to analyse factors influencing water quality and communities on lakes created in a cutaway bog in the Seda Mire, Latvia. The residual peat amount and the lake feeding conditions (the balance between ground water and inflow from adjacent river during the spring season) were found to be major factors influencing aquatic chemistry and studied habitats. The studied cutaway lakes cannot be considered as typical bog lakes regarding hydrochemical composition (especially regarding concentrations of major dissolved ions, $\mathrm{pH}$ and water colour) and hydrobiological features (metabolic activity, biomass and number of species of phytoplankton and zoobenthos). Considering aquatic chemistry and community structure of the cutaway lakes, a recommendation was made in respect to peat excavation to transform peat mining sites to lakes.
\end{abstract}

Key words: biological diversity, cutaway lakes, cutaway peatland, Latvia, water quality.

\section{INTRODUCTION}

Remediation of industrially-milled peatland is an important task in the sustainable management of natural resources. Several approaches for wetland restoration, depending on local climatic and hydrological conditions, topography, physico-chemical properties of peat, as well as peat mining techniques, have been developed worldwide. However, most of the restoration activities include regulation of hydrological regime and surface topography in order to reintroduce typical bog vegetation, especially, Sphagnum cover, and to restore the peatland's ecosystem close to its original conditions (Lamers et al., 2002; Farrell and Doyle, 2003; Gorham and Rochefort, 2003). In many countries, a significant part of peat mines are and will be left as semi-natural wilderness areas, and spontaneous re-vegetation is taking place (Lavoie et al., 2003; Girard et al., 2002). In recent years, creation of shallow lakes (1-2 m depth) in territories of cutaway peatlands is also considered as a valuable management activity. After the cessation of peat harvesting, different areas within a single peat field can vary considerably in terms of depth and type of the residual peat, the underlying soil type and drainage characteristics (McNally, 1998).
Previous studies (Higgins and Colleran, 2004; Lally et al., 2008; Lundin et al., 2008) have indicated that, prior to the creation of cutaway lakes, considerable on-site management work should be carried out. Much of the residual peat substrate usually is removed from the cutaway to create a basin, exposing some of the underlying mineral sub-soil (Higgins and Colleran, 2004), but significant amounts of peat mass from highly decomposed peat layers may be left after major peat mining. Depending on the lake depth, size, aquatic chemistry, vegetation and other properties, the created lakes can be used for recreation purposes or can be important sites for wildlife, especially waterfowl conservation (Lamers et al., 2002).

Peat deposits cover about $10.4 \%$ of the area of Latvia, of which the mined peat area occupies $0.4 \%$ of the country. Currently, about $25 \%$ of the peat deposits prepared for mining are being utilised, and major development of new fields is not expected (Anonymous, 1999). However, management activities for reclamation of cutaway peatlands in Latvia have not been carried out. These territories are abandoned to naturally re-vegetate, or flood if the site lies below the water table. Peat harvesting in the Seda mire started after 
the Second World War and has been very intensive, exhausting much of the peat reserves. Peat harvesting is gradually ceasing, leaving large areas of abandoned cutaway peatland requiring reclamation. A significant part of the area has flooded.

This study was focused on determining various physicochemical and biological parameters in lakes created on cutaway territories in the Seda mire, in order to elucidate the primary factors impacting water quality in the cutaway lakes and to aid future design for optimal water quality.

\section{MATERIALS AND METHODS}

Study area. The Seda mire developed as a result of paludification of land and partially overgrown lakes after the Ice Age. The area of Seda mire is estimated to be 7582 ha, of which 6300 ha are fen, 941 ha is raised bog and 341 ha is transitional bog (Krauklis, 1998). As a result of peat mining, some parts of the mire were transformed to meadows or overgrown by shrubs and later transforming to forest land (Larmanis, 2003).

The climate in area of the Seda mire can be described as wet and comparatively cold, with yearly mean sum of precipitation $650 \mathrm{~mm}$ per year. Mean annual temperature (1950-2003) is $\sim 5.2{ }^{\circ} \mathrm{C}$; mean temperature in January is $-6{ }^{\circ} \mathrm{C}$, and in July is $\sim 17{ }^{\circ} \mathrm{C}$. During the last decades, the temperature in the region of the Seda bog has increased by $0.8{ }^{\circ} \mathrm{C}$, in comparison with the temperature mean for 1961-1990.

Currently, the biggest area of the mire consists of peat extraction fields, including some where peat excavation is ongoing (approximately $3 \mathrm{~km}^{2}$ ). Other parts of the prior fields are overgrown with shrubs or flooded. Average thickness of the peat layer is approximately $3 \mathrm{~m}$, with maximum thickness up to $5 \mathrm{~m}$. Peat resources are estimated at approximately 97.6 millions tons. Industrial peat mining started in 1954 and recently significant parts of the former abandoned peat mining sites have flooded. Channels and dams take up a significant part of the landscape (Larmanis, 2003).

The studied cutaway lakes (sampling stations Nos. 1 and 2) and a lake of natural origin (Lake Seda, sampling station No. 4) are located in the Seda mire in North Vidzeme (Fig. 1). Water depth at sampling station No. 1 was 0.7-1 m. Sediments were composed of peat rich in detrital material. Water depth at sampling station No. 2 in the pelagic zone was $1-1.5 \mathrm{~m}$, and in the littoral zone around $0.5 \mathrm{~m}$. A substantial part of profundal sediments was composed of mineral material, while organic-rich mud dominated in the littoral zone. Both of the cutaway lakes were overgrown by macrophytes (Potamogeton sp., Phragmites sp., Typha sp., Juncus sp., Lemna sp., Nymphaea candida C. Presl., Nuphar lutea). Sampling station No. 3 was located in the River Staklupite, which drains the peat extraction fields. Lake Seda (sampling station No. 4) is a dystrophic brown-water lake of natural origin. The water depth in the pelagic zone was approximately $2 \mathrm{~m}$, and in the littoral $1.5 \mathrm{~m}$. The lake littoral sediments were composed of peat and the profundal sediments were covered by the bryophyte Fontinalis sp. The other natural lakes - L. Tolkovas (sampling station No. 5) and L. Islienas (sampling station No. 6; sampling of water chemistry only) are situated in the Teiči mire (Teiči Nature Reserve). Lake Tolkovas is a typical dystrophic bog lake with low mineralisation, and L. Islienas is a dyseutrophic lake with low mineralization and brown water colour. Water depth in the pelagic zone of L. Tolkovas was $6.5 \mathrm{~m}$ and profundal sediments were composed of loamy mud. Littoral sediments were composed of compact peat, partly covered with Sphagnum sp.

Sampling and laboratory analysis. Samples for hydrochemical analysis were taken in March, June, August and September of 2005 and in March, June and August of 2006. Surface water samples were collected at a depth of $0.5 \mathrm{~m}$ using a Ruttner bathometer. Water temperature and dissolved oxygen were measured on site using an oxymeter (HACH LDO HQ10). Concentrations of nutrients and major

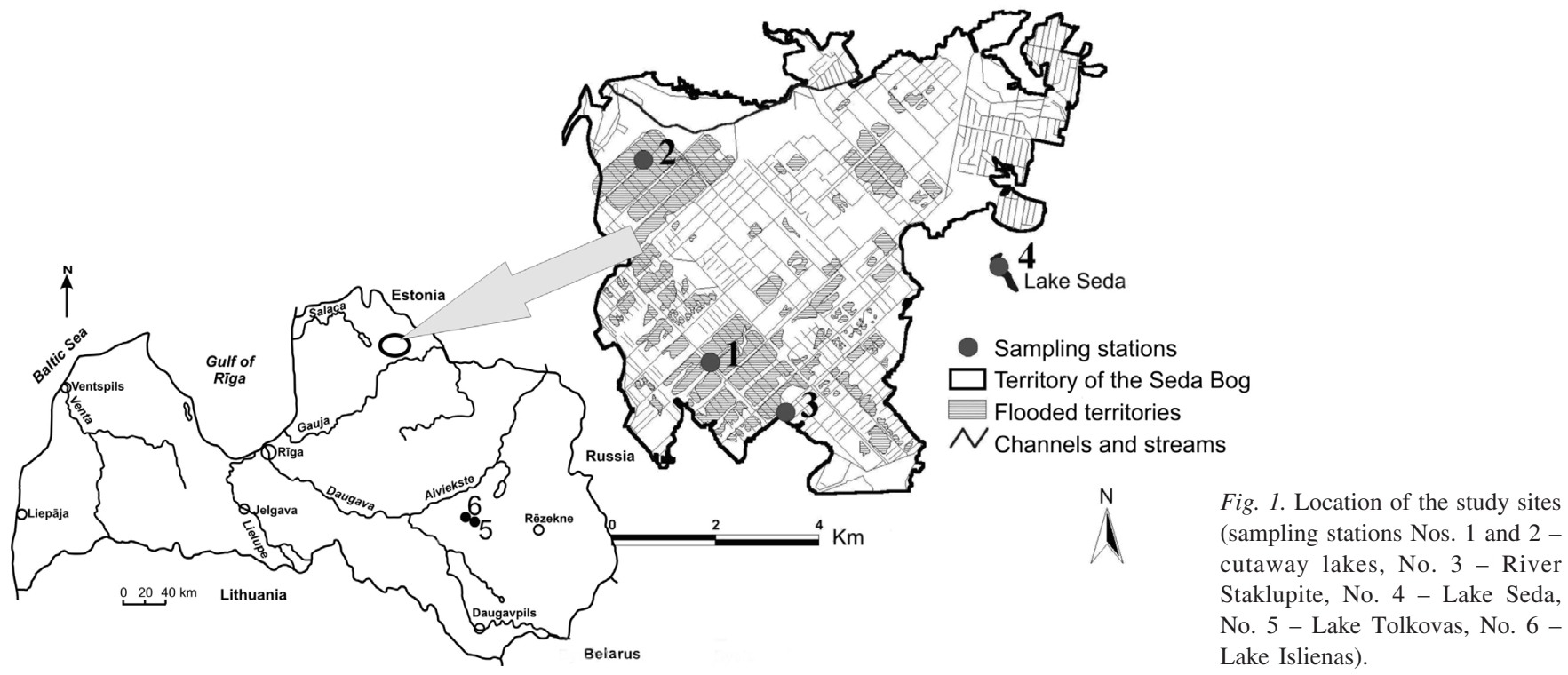


inorganic ions were analysed in the Laboratory of Environmental Quality and Monitoring, University of Latvia. Water samples were delivered to the laboratory and analysed within 24 hours. $\mathrm{pH}$ was measured using a "HACH one $\mathrm{pH}$ meter", conductivity and total dissolved solids were determined using a $\mathrm{HACH}$ conductometer. Colour (true) was measured spectrophotometrically at $455 \mathrm{~nm}$ (Anonymous, 1992). Phosphate ion concentration was determined using the ascorbic acid reduction method with persulphate digestion (Anonymous, 1992) and ammonia by the indophenol method. Phosphate ion, total nitrogen, silica, total iron, nitrate and nitrite concentrations were determined spectrophotometrically using a HACH DR2000 (Anonymous, 1992). Sulphate ion concentration was measured by turbidimetric method. Concentrations of chloride, calcium, bicarbonate ions and total hardness were determined titrimetrically. Chemical oxygen demand was determined using oxidation with $\mathrm{K}_{2} \mathrm{Cr}_{2} \mathrm{O}_{7}$ (Anonymous, 1992).

Sediment samples for analysis of heavy metals were taken in September of 2005 and in June 2006 using an Ekman sediment grabber. Concentrations of heavy metals $(\mathrm{Cd}, \mathrm{Cu}$, $\mathrm{Mn}, \mathrm{Pb}, \mathrm{Zn}, \mathrm{Ni}, \mathrm{Co}, \mathrm{Fe}$ ) in sediments were determined using atomic absorption spectrometry (AAS Perkin-Elmer 403). Analyses were conducted at the Institute of Biology.

Water and sediment samples for bacterial analysis were taken in September 2005. The following groups of bacterioplankton and bacteriobenthos were determined according to methods previously described (Romanenko and Kuznetsov, 1974; Clesceri et al., 1998): aerobic psychrophilic, facultatively anaerobic, oligocarbophylic and cellulose-destroying bacteria. Results were expressed as colony forming units (CFU) per $1 \mathrm{~cm}^{3}$ of water or 1 gram of sediments.

Phytoplankton samples were collected in August and September of 2005 at depth $0.5 \mathrm{~m}$ and preserved with Lugol solution. Phytoplankton cells were identified, measured and counted with a Leica DML inverted microscope. Biovolumes were calculated by comparing cells to simple shapes and applying standard geometric formulae (Utermohl, 1958).

Samples for zooplankton analysis were collected in August and September of 2005. $100 \mathrm{dm}^{3}$ of water were filtered through an Apstein net $(64 \mu \mathrm{m})$ and zooplankton samples were preserved with $40 \%$ formalin solution. Quantitative and qualitative analyses were conducted on $1 \mathrm{~cm}^{3}$ subsamples under a Carl Zeiss Jena microscope. Biomasses were calculated by comparing organisms to simple shapes and applying standard geometric formulae (Bottrell et al., 1976).

Samples of benthic macroinvertebrates were collected in September of 2005 from the profundal zone using an Ekman sediment grabber (opening of $20 \times 20 \mathrm{~cm}$ ). The number of organisms per square meter was calculated and their biomass was estimated. Macroinvertebrates were categorised according to Family or Order.
Data from sampling stations Nos. 1, 2 and 4 were compared with mean values (1997-2003 and 2007) from sampling stations Nos. 5 and 6. Water quality data were analysed using the programmes SPSS 14.0 and AquaChem 3.70. Comparison of sampling sites was performed using one way analysis of variance (ANOVA) followed by a Tukey HSD test as post hoc procedure at $P \leq 0.05$.

\section{RESULTS}

Major differences in the aquatic chemistry of the studied lakes were found (Table 1, Figs. 2 and 3). Both cutaway and natural lakes of Seda mire had similar pH levels (6.927.43), moderately high conductivity (145.9-286.8 $\mu \mathrm{S} \mathrm{cm}^{-1}$ ) and concentrations of dissolved inorganic ions as well as moderately high colour. The dystrophic L. Tolkovas (sampling station No. 5) was acidic ( $\mathrm{pH} 4.44$ ), with low concentrations of dissolved inorganic ions and very low water colour $\left(97{ }^{\circ} \mathrm{Pt} / \mathrm{Co}\right)$, which is unusual for dystrophic lakes (Table 1). The dyseutrophic L. Islienas has very high water colour ( $\left.>500{ }^{\circ} \mathrm{Pt} / \mathrm{Co}\right)$, but comparatively higher $\mathrm{pH}$ (5.82). Dissolved oxygen levels were similar in all four waterbodies of the Seda mire and followed typical seasonal trends, with higher concentrations in summer and low concentrations (up to $0.18 \mathrm{mg} \mathrm{dm}^{-3}$ at sampling station 1) during winter and early spring (Fig. 2).

Concentration of total dissolved solids in cutaway lakes of the Seda mire was higher than in the studied natural dystrophic lakes. Both cutaway and natural lakes of Seda mire belong to the $\mathrm{Ca}-\mathrm{Mg}-\mathrm{HCO}_{3}$ group, while L. Tolkovas (sampling station No. 5) has relatively higher concentrations of chloride, bicarbonate and sulphate ions, and therefore, belongs to the $\mathrm{Cl}-\mathrm{HCO}_{3}-\mathrm{SO}_{4}$ group. Piper diagrams (Piper 1944), which can be used to characterise different water types, show that both typical surface waters of Latvia (Lakes Burtnieks, Dridzis, Cieceres and Engures) and waterbodies of the Seda mire have elevated temporary hardness due to increased $\mathrm{Ca}^{2+}, \mathrm{Mg}^{2+}$ and $\mathrm{HCO}_{3}{ }^{-}$concentrations (Fig. 4). Bog lakes with low mineralisation have a relatively increased the proportion of sulphate and chloride ions.

Concentrations of heavy metals in the cutaway lake sediments are lower than in dystrophic lakes, as they have not been influenced by pollution (Fig. 5).

The bacterioplankton and bacteriobenthos of the cutaway lakes were dominated by oligocarbophylic and cellulosedegrading bacteria, while saprophytes typically dominated in the natural lakes. However, this dominance was comparatively more expressed at sampling station No. 2, shown by the CFU both in bacterioplankton and bacteriobenthos (Table 2).

Phytoplankton samples at sampling station No. 1 contained a low number of taxa and low phytoplankton biomass $\left(0.12-0.18 \mathrm{mg} \mathrm{dm}^{-3}\right)$. Phytoplankton was represented by Cyanophyceae, Euglenophyceae, Dinophyceae, Cryptomo- 
CHEMICAL COMPOSITION OF THE STUDIED LAKES*

\begin{tabular}{|c|c|c|c|c|c|c|}
\hline Parameter & $\begin{array}{c}\text { Sampling station } \\
\text { No. } 1 \\
\end{array}$ & $\begin{array}{c}\text { Sampling station } \\
\text { No. } 2 \\
\end{array}$ & $\begin{array}{c}\text { Sampling station } \\
\text { No. } 3\end{array}$ & $\begin{array}{c}\text { Sampling station } \\
\text { No. } 4\end{array}$ & $\begin{array}{c}\text { Sampling station } \\
\text { No. } 5\end{array}$ & $\begin{array}{c}\text { Sampling station } \\
\text { No. } 6\end{array}$ \\
\hline $\mathrm{PO}_{4}^{3-}, \mathrm{mg} \mathrm{dm}^{-3}$ & $\begin{array}{c}0.033( \pm 0.03) \mathrm{ab} \\
7\end{array}$ & $\begin{array}{c}0.022( \pm 0.015) \mathrm{a} \\
7\end{array}$ & $\begin{array}{c}0.029( \pm 0.012) \mathrm{a} \\
6\end{array}$ & $\begin{array}{c}0.064( \pm 0.014) \mathrm{b} \\
6\end{array}$ & $\begin{array}{c}0.015( \pm 0.015) \mathrm{a} \\
7\end{array}$ & $\begin{array}{c}0.031( \pm 0.014) \mathrm{a} \\
7\end{array}$ \\
\hline$\underset{n}{\mathrm{~N}-\mathrm{NH}_{4}}{ }^{+}, \mathrm{mg} \mathrm{dm}^{-3}$ & $\begin{array}{c}0.65( \pm 0.49) \mathrm{ab} \\
7\end{array}$ & $\begin{array}{c}0.32( \pm 0.07) \mathrm{a} \\
7\end{array}$ & $\begin{array}{c}0.48( \pm 0.10) \mathrm{a} \\
7\end{array}$ & $\begin{array}{c}0.71( \pm 0.19) \mathrm{ab} \\
7\end{array}$ & $\begin{array}{c}0.32( \pm 0.10) \mathrm{a} \\
7\end{array}$ & $\begin{array}{c}1.03( \pm 0.31) \mathrm{b} \\
7\end{array}$ \\
\hline$\underset{n}{\mathrm{~N}-\mathrm{NO}_{2}{ }^{-} \mathrm{mg} \mathrm{dm}^{-3}}$ & $\begin{array}{c}0.014( \pm 0.006) \mathrm{a} \\
7\end{array}$ & $\begin{array}{c}0.009( \pm 0.003) \mathrm{a} \\
7\end{array}$ & $\begin{array}{c}0.013( \pm 0.004) \mathrm{a} \\
7\end{array}$ & $\begin{array}{c}0.024( \pm 0.007) \mathrm{b} \\
7\end{array}$ & $0.010( \pm 0.003) \mathrm{a}$ & $\begin{array}{c}0.032( \pm 0.007) \mathrm{b} \\
7\end{array}$ \\
\hline $\mathrm{Si}_{\text {tot }}, \mathrm{mg} \mathrm{dm}^{-3}$ & $\begin{array}{c}4.93( \pm 2.82) \mathrm{b} \\
7\end{array}$ & $\begin{array}{c}2.41( \pm 0.79) \mathrm{ab} \\
7\end{array}$ & $\begin{array}{c}5.00( \pm 2.92) \mathrm{b} \\
7\end{array}$ & $\begin{array}{c}5.64( \pm 2.99) b \\
7\end{array}$ & $\begin{array}{c}1.27( \pm 0.64) \mathrm{a} \\
7\end{array}$ & $\begin{array}{c}2.50( \pm 1.96) \mathrm{ab} \\
7\end{array}$ \\
\hline $\mathrm{Fe}_{n} \mathrm{tot}, \mathrm{mg} \mathrm{dm}^{-3}$ & $\begin{array}{c}1.58( \pm 0.91) \mathrm{a} \\
7\end{array}$ & $\begin{array}{c}0.48( \pm 0.24) \mathrm{a} \\
7\end{array}$ & $\begin{array}{c}1.87( \pm 1.47) \mathrm{a} \\
7\end{array}$ & $\begin{array}{c}5.01( \pm 2.23) \mathrm{b} \\
7\end{array}$ & $\begin{array}{c}0.32( \pm 0.09) \mathrm{a} \\
7\end{array}$ & $\begin{array}{c}1.22( \pm 0.49) \mathrm{a} \\
7\end{array}$ \\
\hline $\begin{array}{l}\mathrm{pH} \\
n\end{array}$ & $\begin{array}{c}7.33( \pm 0.45) \mathrm{c} \\
7\end{array}$ & $\begin{array}{c}7.43( \pm 0.43) \mathrm{c} \\
7\end{array}$ & $\begin{array}{c}7.15( \pm 0.32) \mathrm{c} \\
7\end{array}$ & $\begin{array}{c}6.92( \pm 0.60) \mathrm{c} \\
7\end{array}$ & $\begin{array}{c}4.44( \pm 0.15) \mathrm{a} \\
7\end{array}$ & $\begin{array}{c}5.82( \pm 0.98) \mathrm{b} \\
7\end{array}$ \\
\hline $\begin{array}{l}\text { Colour, }{ }^{0} \mathrm{Pt} / \mathrm{Co} \\
\end{array}$ & $\begin{array}{c}187( \pm 60) \mathrm{a} \\
7\end{array}$ & $\begin{array}{c}108( \pm 35) \mathrm{a} \\
7\end{array}$ & $\begin{array}{c}236( \pm 72) \mathrm{a} \\
7\end{array}$ & $\begin{array}{c}562( \pm 201) b \\
7\end{array}$ & $\begin{array}{c}97( \pm 38) \mathrm{a} \\
7\end{array}$ & $\begin{array}{c}537( \pm 132) \mathrm{b} \\
7\end{array}$ \\
\hline $\begin{array}{l}\mathrm{COD}, \mathrm{mg} \mathrm{dm}^{-3} \\
\text {. }\end{array}$ & $\begin{array}{c}39.57( \pm 15.67) \mathrm{ab} \\
7\end{array}$ & $\begin{array}{c}45.57( \pm 11.93) \mathrm{abc} \\
7\end{array}$ & $\begin{array}{c}54.71( \pm 17.55) b c \\
7\end{array}$ & $\begin{array}{c}76.43( \pm 35.01) \mathrm{cd} \\
7\end{array}$ & $\begin{array}{c}15.86( \pm 6.20) \mathrm{a} \\
7\end{array}$ & $\begin{array}{c}92.43( \pm 18.54) \mathrm{d} \\
7\end{array}$ \\
\hline $\begin{array}{l}\text { Cond, } \mu \mathrm{S} \mathrm{cm}^{-1} \\
n\end{array}$ & $\begin{array}{c}286.8( \pm 87.5) \mathrm{d} \\
6\end{array}$ & $\begin{array}{c}226.8( \pm 31.2) \mathrm{cd} \\
6\end{array}$ & $\begin{array}{c}216.0( \pm 53.5) \mathrm{cd} \\
6\end{array}$ & $\begin{array}{c}145.9( \pm 56.4) b c \\
6\end{array}$ & $\begin{array}{c}25.0( \pm 3.6) \mathrm{a} \\
7\end{array}$ & $\begin{array}{c}72.8( \pm 38.0) \mathrm{ab} \\
7\end{array}$ \\
\hline$\underset{n}{\mathrm{HCO}_{3}}{ }^{-}, \mathrm{mg} \mathrm{dm}^{-3}$ & $\begin{array}{c}191.7( \pm 62.0) \mathrm{d} \\
7\end{array}$ & $\begin{array}{c}149.0( \pm 23.6) \mathrm{cd} \\
7\end{array}$ & $\begin{array}{c}161.2( \pm 57.4) \mathrm{d} \\
7\end{array}$ & $\begin{array}{c}95.9( \pm 34.8) \mathrm{bc} \\
7\end{array}$ & $\begin{array}{c}18.3( \pm 6.1) \mathrm{a} \\
7\end{array}$ & $\begin{array}{c}47.1( \pm 18.9) \mathrm{a} \\
7\end{array}$ \\
\hline$\underset{n}{\mathrm{SO}_{4}{ }^{2-}, \mathrm{mg} \mathrm{dm}^{-3}}$ & $\begin{array}{c}8.28( \pm 0.95) \mathrm{a} \\
7\end{array}$ & $\begin{array}{c}8.00( \pm 0.82) \mathrm{a} \\
7\end{array}$ & $\begin{array}{c}10.86( \pm 3.44) \mathrm{a} \\
7\end{array}$ & $\begin{array}{c}18.00( \pm 5.60) \mathrm{b} \\
7\end{array}$ & $\begin{array}{c}8.14( \pm 2.27) \mathrm{a} \\
7\end{array}$ & $\begin{array}{c}18.71( \pm 4.03) \mathrm{b} \\
7\end{array}$ \\
\hline$\underset{n}{\mathrm{Cl}^{-}, \mathrm{mg} \mathrm{dm}^{-3}}$ & $\begin{array}{c}9.47( \pm 2.39) \\
7\end{array}$ & $\begin{array}{c}9.75( \pm 1.75) \\
7\end{array}$ & $\begin{array}{c}8.81( \pm 1.81) \\
7\end{array}$ & $\begin{array}{c}9.13( \pm 1.91) \\
7\end{array}$ & $\begin{array}{c}15.67( \pm 10.60) \\
7\end{array}$ & $\begin{array}{c}16.63( \pm 13.81) \\
7\end{array}$ \\
\hline $\mathrm{Ca}_{n}^{2+}, \mathrm{mg} \mathrm{dm}^{-3}$ & $\begin{array}{c}43.91( \pm 13.93) \mathrm{d} \\
7\end{array}$ & $\begin{array}{c}34.57( \pm 4.49) \mathrm{cd} \\
7\end{array}$ & $\begin{array}{c}39.42( \pm 12.48) \mathrm{d} \\
7\end{array}$ & $\begin{array}{c}23.28( \pm 3.66) \mathrm{bc} \\
7\end{array}$ & $\begin{array}{c}3.09( \pm 3.44) \mathrm{a} \\
7\end{array}$ & $\begin{array}{c}10.26( \pm 5.40) \mathrm{ab} \\
7\end{array}$ \\
\hline $\mathrm{Mg}_{n}^{2+}, \mathrm{mg} \mathrm{dm}^{-3}$ & $\begin{array}{c}12.10( \pm 3.13) \mathrm{b} \\
7\end{array}$ & $\begin{array}{c}11.99( \pm 3.39) \mathrm{b} \\
7\end{array}$ & $\begin{array}{c}11.53( \pm 6.93) \mathrm{b} \\
7\end{array}$ & $\begin{array}{c}7.96( \pm 5.01) \mathrm{ab} \\
7\end{array}$ & $1.87\left(\begin{array}{c}( \pm 2.44) \mathrm{a} \\
7\end{array}\right.$ & $\begin{array}{c}6.65( \pm 7.39) \mathrm{ab} \\
7\end{array}$ \\
\hline $\mathrm{K}_{n}^{+}, \mathrm{mg} \mathrm{dm}^{-3}$ & $\begin{array}{c}0.93( \pm 0.45) \\
3\end{array}$ & $\begin{array}{c}1.77( \pm 0.65) \\
3\end{array}$ & $\begin{array}{c}1.00( \pm 0.26) \\
3\end{array}$ & $\begin{array}{c}0.90( \pm 0.63) \\
3\end{array}$ & $\begin{array}{c}0.70( \pm 0.21) \\
5\end{array}$ & $\begin{array}{c}1.06( \pm 0.77) \\
5\end{array}$ \\
\hline
\end{tabular}

$\overline{* \text { mean } \pm \text { S.D., } n}-$ number of samples. Different letters within a row indicate significantly different groups in Tukey HSD test at $P \leq 0.05$

Sampling station No 2

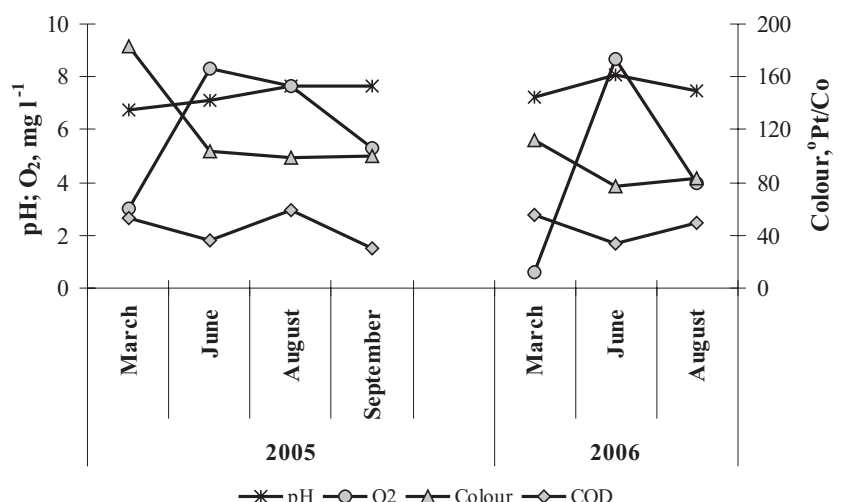

Fig. 2. Seasonal changes of $\mathrm{pH}$, dissolved oxygen, water colour and chemical oxygen demand at sampling station No. 2.

nadineae, Chrysophyceae, and Bacillariophyceae. Phytoplankton samples had a high number of taxa, but low biomass $\left(0.12-0.22 \mathrm{mg} \mathrm{dm}^{-3}\right)$, in sampling station No. 2, where phytoplankton was represented by eight algae divi-

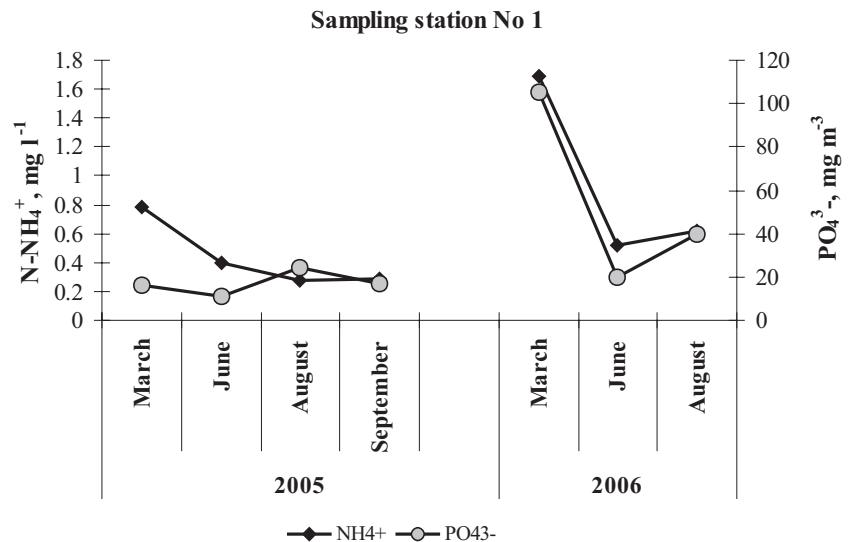

Fig. 3. Seasonal changes in ammonium and phosphate ion concentration at sampling station No. 1.

sions: Cyanophyceae, Cryptophyceae, Chrysophyceae, Euglenophyceae, Dinophyceae, Raphidophyceae, Bacillariophyceae, and Chlorophyceae (Table 3). Phytoplankton at sampling station No. 4 was practically dominated by 


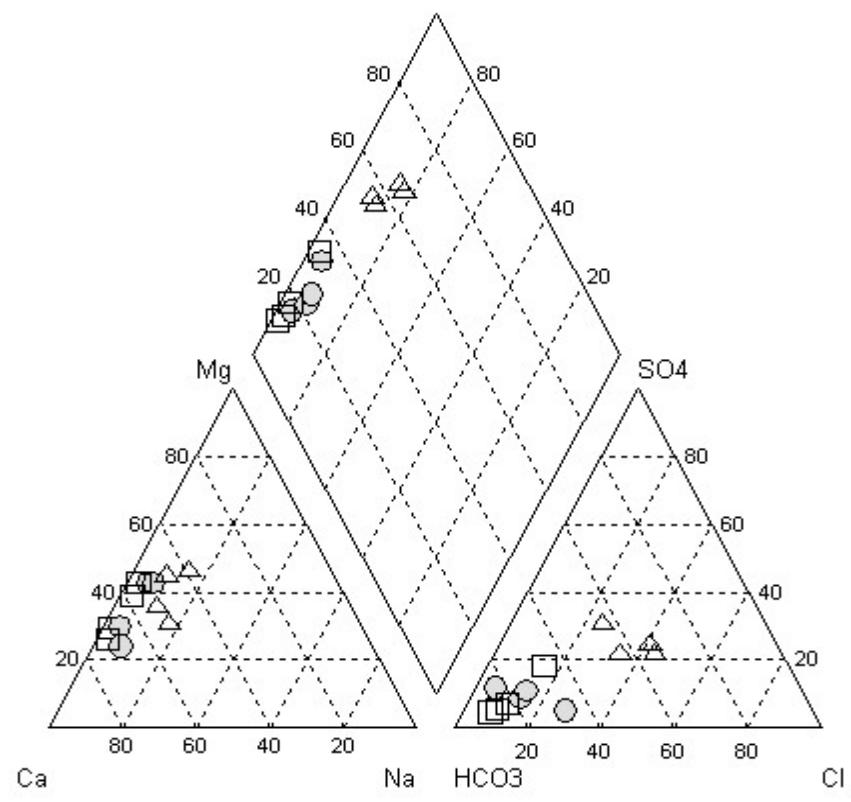

Fig. 4. Piper diagram (O - Lakes Burtnieks, Dridzis, Cieceres and Engures; $\triangle$ - bog lakes: Siksalas, Tolkovas, Lielezers, Islienas; $\square$ - lakes of Seda Bog).

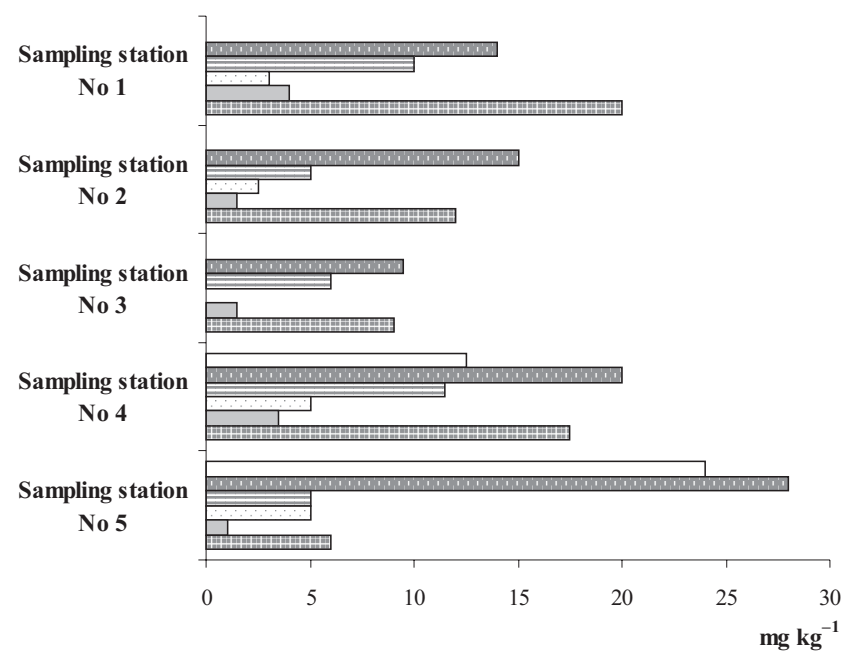

$\square \mathrm{Pb} \square \mathrm{Zn}$ 目i $\square \mathrm{Cu} \square \mathrm{Cd} \square \mathrm{Co}$

Fig. 5. Concentrations of heavy metals in sediments.
NUMBER OF AEROBIC SAPROPHYTES ( $\mathrm{SB}_{\mathrm{aer}}$ ), ANAEROBIC SAPROPHYTES ( $\left(\mathrm{SB}_{\text {anaer }}\right)$, OLIGOCARBOPHYLIC (OB) AND CELLULOSE-DESTROYING (CB) BACTERIA IN THE STUDIED WATERBODIES

\begin{tabular}{|c|c|c|c|c|c|c|c|c|}
\hline \multirow{2}{*}{$\begin{array}{c}\text { Sampling } \\
\text { station }\end{array}$} & \multicolumn{4}{|c|}{ Bacterioplankton $\left.(\mathrm{CFU} \mathrm{cm})^{-3}\right)$} & \multicolumn{4}{|c|}{ Bacteriobenthos $\left(\mathrm{CFU} \mathrm{g}{ }^{-1}\right)$} \\
\hline & $\mathrm{SB}_{\mathrm{aer}}$ & $\mathrm{SB}_{\text {anae }}$ & OB & $\mathrm{CB}$ & $\mathrm{SB}_{\text {aer }}$ & $\mathrm{SB}_{\text {anaer }}$ & $\mathrm{OB}$ & $\mathrm{CB}$ \\
\hline No. 1 & 690 & 90 & 870 & 700 & 4000 & 1750 & 7250 & 9250 \\
\hline No. 2 & 720 & 390 & 3650 & 4000 & 15750 & 485000 & 50000 & 26250 \\
\hline No. 4 & 240 & 200 & 960 & 1300 & 4000 & 3000 & 11250 & 9250 \\
\hline No. 5 & 460 & - & - & - & 4470 & - & - & - \\
\hline $\begin{array}{l}\text { Dyseutro- } \\
\text { phic lakes }\end{array}$ & 2320 & - & - & - & 131651 & - & - & - \\
\hline
\end{tabular}

Raphidophyceae, particularly by Gonyostomum semen, which is typical for dystrophic lakes, with low numbers of Chrysophyceae, Cryptomonadineae, Euglenophyceae and Bacillariophyceae. Total biomass at this sampling station was comparatively high (2.4-4.9 $\mathrm{mg} \mathrm{dm}^{-3}$ ) in August and September. In September, there was a decrease in the biomass of blue-green algae at sampling station No. 3 .

Zooplankton abundance was higher in September than in August. At sampling station No. 1, a comparatively low number of individuals (28.0-32.2 indiv. $\mathrm{dm}^{-3}$ ) and high biomass (179.56 $\mathrm{mg} \mathrm{dm}^{-3}$ ) of zooplankton were observed due to large-bodied Cladocera (for example, Acroperus harpae and Graptoloberis testudinaria). Among the Rotifera, Keratella cochlearis and Polyarthra sp. dominated. At sampling station No. 2, Rotifera was the largest group, dominated by Synchaeta sp., followed by Asplanchna priodonta/henrietta, Lecane sp., Keratella cochlearis, Keratella quadrata, and Polyarthra sp. The zooplankton community at sampling station No. 4 (natural L. Seda) was dominated by Asplanchna priodonta/Henrietta, followed by Polyarthra sp., Keratella cochlearis, Conochiloides sp. and the cladocerans Bosmina longirostris, Ceriodaphnia quadrangula, and Chydorus sphaericus, similar to the zooplankton of dyseutrophic lakes in Teiči Nature Reserve.

Considering high habitat heterogeneity in the littoral zone, for the analyses of benthic macroinvertebrates, only samples from the profundal zone were chosen.

Table 3

PHYTOPLANKTON BIOMASS $\left(\mathrm{mg} \mathrm{dm}^{-3}\right.$ ) IN AUGUST AND SEPTEMBER OF 2005

\begin{tabular}{l|c|c|c|c|c|c|c|c|c}
\hline & $\begin{array}{c}\text { Cyano- } \\
\text { phyceae }\end{array}$ & $\begin{array}{c}\text { Chryso- } \\
\text { phyceae }\end{array}$ & $\begin{array}{c}\text { Crypto- } \\
\text { phyceae }\end{array}$ & $\begin{array}{c}\text { Eugleno- } \\
\text { phyceae }\end{array}$ & $\begin{array}{c}\text { Dino- } \\
\text { phyceae }\end{array}$ & $\begin{array}{c}\text { Bacillario- } \\
\text { phyceae }\end{array}$ & $\begin{array}{c}\text { Chloro- } \\
\text { phyceae }\end{array}$ & $\begin{array}{c}\text { Chloromonado- } \\
\text { phyceae }\end{array}$ & $\begin{array}{c}\text { Total } \\
\text { Station No. 1, August }\end{array}$ \\
\hline & 0.001 & 0.097 & 0.002 & 0.003 & 0.072 & 0.003 & 0.001 & 0.179 \\
Station No. 1, September & & 0.084 & 0.001 & 0.002 & 0.036 & 0.001 & 0.124 \\
Station No. 2, August & 0.057 & 0.074 & 0.002 & 0.044 & 0.028 & 0.008 & 0.007 & 0.003 & 0.223 \\
Station No. 2, September & 0.022 & 0.014 & 0.004 & 0.037 & 0.017 & 0.006 & 0.008 & 0.015 & 0.123 \\
Station No. 3, August & 0.002 & 0.001 & 0.034 & & 0.001 & 0.001 & & 0.039 \\
Station No. 3, September & 0.004 & 0.001 & 0.049 & 0.001 & 0.001 & & & 0.056 \\
Station No. 4, August & & 0.003 & 0.001 & 0.002 & & 0.002 & 0.006 & 4.874 & 4.888 \\
Station No. 4, September & & 0.001 & & 0.001 & & & & 2.372 & 2.374
\end{tabular}


The highest number of taxa was found at sampling station No. 2 (18 taxa), lower at stations No. 1 and No. 4 (9 taxa) and the lowest at No. 5 (6 taxa).

The highest density of benthic macroinvertebrates was found at sampling station No. 4 (41 700 indiv. $\mathrm{m}^{-2}$ ), mainly due to Oligochaete abundance. Lower density was characteristic for sampling station No. 2 (7200 indiv. $\mathrm{m}^{-2}$ ), and the lowest at station No. 5 (2100 indiv. $\mathrm{m}^{-2}$ ).

The highest biomass was observed at sampling station No. 2 $\left(15 \mathrm{~g} \mathrm{~m}^{-2}\right)$, mainly composed of herbivorous snails, larvae of insects (mayflies Ephemeroptera) and filterers - mussels Sphaeridae. Slightly lower biomass was found at the reference sampling station No. $4\left(12 \mathrm{~g} \mathrm{~m}^{-2}\right)$, mainly composed of sediment feeders Oligochaetes, snails and detritivorous larvae of non-biting midges Chironomidae (Fig. 6). Low biomass $\left(6 \mathrm{~g} \mathrm{~m}^{-2}\right)$ was characteristic for L. Tolkovas (station No. 5). The lowest biomass was found at sampling station No. $1\left(2 \mathrm{~g} \mathrm{~m}^{-2}\right)$.

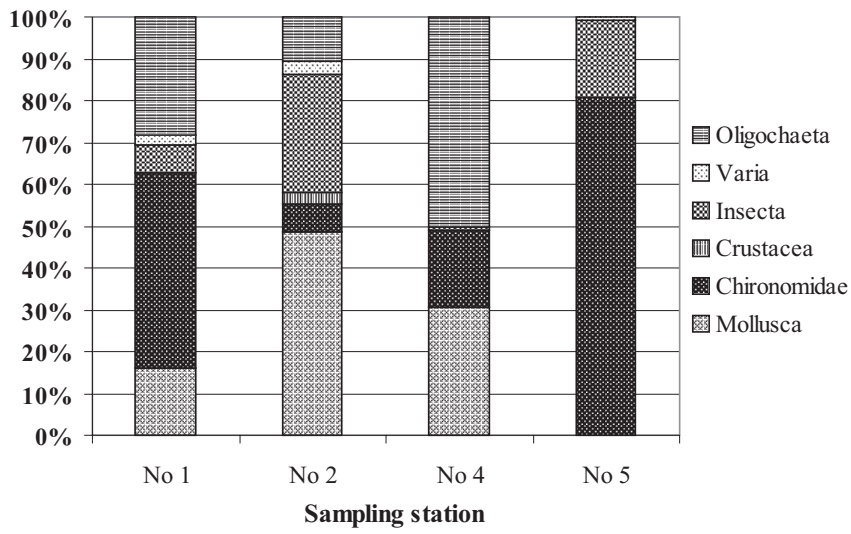

Fig. 6. Percentage of dominating taxa (\%) of total macroinvertebrate biomass $\left(\mathrm{g} \mathrm{m}^{-2}\right)$ in the profundal zone.

\section{DISCUSSION}

The studied cutaway lakes differ in physico-chemical characteristics and nutrient concentrations (Table 1). The studied cutaway lakes and the River Stakḷupite have significantly $(P \leq 0.05)$ higher $\mathrm{pH}$, conductivity and concentrations of $\mathrm{HCO}_{3}{ }^{-}, \mathrm{Ca}^{2+}, \mathrm{Mg}^{2+}$. These differences might be related to the diverse underlying sediments and major differences in hydrological regimes, as studies on artificial cutaway lakes in other countries suggest (Higgins and Colleran, 2004; Lundin et al., 2008). In some areas in the Seda mire, the peat layer left after peat extraction was insufficient to prevent the influence from mineral soil and ground water. This might be a possible explanation for lower water colour observed in the cutaway lakes compared to brown-water lakes (sampling stations No. 4 and No. 6). During the 1980s, some areas of the Seda mire were ploughed, thus increasing the rate of mineralisation, or even were fertilised with the aim to establish more productive grassland. Due to the economical crisis, these activities ceased in the early 1990s. Mineral rich waters from the
River Seda flooded areas in the northern part of the mire during spring. As a result, the chemical composition characteristic for a mesotrophic lake has developed. This trophic state is characteristic for the cutaway lakes (stations No. 1 and No. 2), where water is alkaline, with high concentrations of calcium, magnesium and other inorganic ions. In these lakes the water buffering capacity is high, but colour values are low as well as phosphorus concentrations. In previously studied dystrophic bog lakes (Urtane and Klavins, 1995; Klavins et al., 2003), much higher values of water colour (200-800 $\left.{ }^{\circ} \mathrm{Pt} / \mathrm{Co}\right)$ have been found. The large standard deviations in chemical parameters of the studied lakes (Table 1) can be explained by seasonal variations in water composition, with higher $\mathrm{pH}$ values and dissolved oxygen levels and lower nutrient concentrations during the summer growing season (Figs. 2 and 3). Aquatic chemistry in these lakes influences primary production, causing relatively low phytoplankton biomass. Presently, the vegetation of the area is characterised by dense macrophyte stands covering up to $40 \%$ of the total water surface area, dominated by reed and bobtail (Phragmites and Typha sp) stands in the emergent waterplant zone and pondweeds Potamogeton natans and Potamogeton zosterifolius dominating in floating-leaved and submerged aquatic plant zones.

The aquatic chemistry and community structure in the two newly developed lakes much differ in comparison with the natural L. Seda (station No. 4). In L. Seda, the littoral sediments were composed of peat, as is common for natural humic lakes, and profundal sediments were covered by the bryophyte Fontinalis sp. This lake had low levels of dissolved ions, inorganic carbon, silica, and nitrogen concentrations. However, phosphate concentrations were higher in comparison with typical phosphate concentrations in lakes of Latvia (Klavins et al., 2002). It could be supposed that elevated concentrations of phosphates are the reason for high phytoplankton biomass in sampling station No. 4. However, the very high biomass in station No. 4 was comprised only by Gonyostomum semen, which occurs in large quantities in brown-water lakes in Northern Europe (Hongve et al., 1987; Cronberg et al., 1988; Lepistö et al., 1994). During the last decades, G. semen has been found in dystrophic and dyseutrophic lakes in Teiči Nature Reserve, in lakes around Riga, and in the Eastern part of Latvia (Druvietis, 2007). The invasion of G. semen might be explained by expansion of its distribution, occupying new lake types, intensive biomass development in several lakes, anthropogenic impact, or more intensive investigation of small lakes (Hörnström, 2002; Findlay et al., 2005).

The trophic status of lakes is associated with nutrient content, which has significant impact on species diversity, richness and seasonal population dynamics (Lindstrom, 2000, Kent et al., 2004; Tadonleke et al., 2005). The main trophic link between chemical components and biota in waterbodies is via the bacterial component. The nutrient content in lakes, at least indirectly, can influence the structure of the bacterioplankton community (Lindstrom, 2000). In humic lakes bacterial development is largely determined by humic sub- 
stances. An increased level of humic substances increases bacterial abundance in humic lakes (Vrede et al., 2003). Autochthonous bacteria utilise and transform aquatic humic substances (HS), decreasing particle size and aromaticity, aliphatic carbon in HS, and increasing the nitrogen content of HS, probably due to some constituents of microbial biomass, such as proteins and amino sugars (Hertkorn et al., 2002). Reduced humic substances (HS) can be reoxidised by anaerobic bacteria, and in general microbial oxidation of HS is a ubiquitous metabolism in the environment (Coates et al., 2002). Simultaneously, decrease in bacterioplankton is caused by consumption by grazers, mainly flagellates (Kent et al., 2004).

In L. Seda, counts of typical saprophytic bacteria were low, which may reflect the low concentration of easily available organic matter. Communities of cellulose destroyers were comparatively more developed. The deeper cutaway lake (station No. 2) had higher bacterial numbers both of saprophytic, and especially oligocarbophylic and cellulose destroying bacteria than those in station No. 1 and L. Seda (station No. 4). In sampling station No. 2, the bacterial processes are likely more active, and consequently also the pelagic food web. Generally, a large proportion of particleassociated bacteria are metabolically active, while only a small fraction of free-living pelagic bacteria are metabolically active (Haglund et al., 2002). We can assume that bacteria in the sediments are more dependent than pelagic forms on HS. Bacteriobenthos counts were low at sampling stations No. 4 and No. 1, but were comparatively high at sampling station No. 2, especially for oligocarbophylic and facultatively anaerobic saprophytic bacteria. Composition of bacterioplankton and bacteriobenthos communities suggest that cycling of organic matter, both in the water column and sediments, was more intense at sampling station No. 2 , compared to that in other lakes.

A comparison of the studied waterbodies in the Seda mire (sampling stations Nos. 1-3) with the natural dystrophic lakes in Teiči Nature Reserve confirmed that, in general, bacterial counts in the Seda territory are low, and accordingly metabolic activity is also low.

The high number of taxa and biomass of macrozoobenthos at sampling station No. 2 might be explained by diverse microhabitat composition and favourable feeding conditions for the macroinvertebrates, provided by the mineral friable sand sediments with a thin mud layer and patchy macrophyte stands. These conditions contrasted with the cutaway lake sampling station No. 1, which contained a deep layer of peat.

Macroinvertebrate communities in both natural lakes (sampling stations Nos. 4 and 5) differed. Lake Tolkovas (station No. 5) is a typical dystrophic lake. Species richness characteristically is low in small humic lakes with simple bottom structure and low abundance of aquatic plants (Heino, 2000). The low biomass in L. Tolkovas $\left(6 \mathrm{~g} \mathrm{~m}^{-2}\right)$ may be associated with the low $\mathrm{pH}$. A limited number of species of Hemiptera, Coleoptera, Trichoptera, Odonata and Diptera are typically present in lakes with low pH (Ward, 1992). The bottom structure of L. Sedas is heterogenous, and peat sediments are covered with Fontinalis sp. stands, rich in Oligochaeta populations. Nutrient content and abundance of food is an important factor for growth of oligochaetes. Growth rates of aquatic oligochaetes feeding on algae are expected to increase with increased $\mathrm{N}$ and $\mathrm{P}$ availability in the surface water, whereas detritivorous oligochaetes, which feed on dead organic matter, fungi and bacteria, are expected to increase with a higher nutrient content and decomposition rate of dead organic matter (Van Duinen et al., 2006).

Gradual accumulation of nutrients in the form of plant detritus and the spread of macrophytes are ongoing processes in shallow parts of the water bodies. In the future, the development of marshes may take place. Newly formed habitats in flooded peat harvest fields could be transformed into important bird nesting and resting locations. Lakes created on cutaway peatlands also could be used for recreation purposes (fishing, bird-watching).

In conclusion, the studied cutaway lakes can not be considered as typical dystrophic or humic lakes, regarding hydrochemical and hydrobiological features, due to diversity of underlying sediments as well as major differences in hydrological regimes. Waters of cutaway lakes are alkaline, with significantly $(P \leq 0.05)$ higher concentrations of calcium, magnesium and bicarbonate ions as well as higher $\mathrm{pH}$ level, but lower water colour, than those in typical brown-water lakes. A comparison of the cutaway lakes in the Seda mire with natural dystrophic lakes confirmed that, in general, bacterial counts in the Seda territory are low, and accordingly, metabolic activity is also low. Number of taxa and biomasses of macrozoobenthos in cutaway lakes differ, mainly due to microhabitat composition (sediment properties and vegetation). In the nearest future, the rehabilitation or reclamation aim — natural regeneration or managed activities - for cutaway peatlands should be defined, planned and managed.

\section{REFERENCES}

Anonymous (1992). HACH Water Analysis Handbook. 2nd ed. Loveland: $\mathrm{HACH}$.

Anonymous (1999). National Programme on Biological Diversity. Riga: Ministry of Environmental Protection and Regional Development.

Bottrell, H.H., Duncan, A., Gliwicz, Z.M., Grygierek, E., Herzig, A., Hillbricht-Ilkowska, A., Kurasawa, H., Larsson, P., Weglenska, T. (1976). A review of some problems in zooplankton production studies. Norw. J. Zool., 24, 419-456.

Clesceri, L.S., Greenberg, A.E., Eaton, A.D. [Eds] (1998). Standard Methods for the Examination of Water and Waste Water. $20^{\text {th }}$ edn.Washington DC: American Public Health Association, American Water Work Association, Water Environment Federation.

Coates, J.D., Cole, K.A., Chakraborty, R., O'Connor, S.M., Achenbach, L.A. (2002). Diversity and ubiquity of bacteria capable of utilizing humic substances as electron donors for anaerobic respiration. Appl. Environ. Microbiol., 68(5), 2445-2452. 
Cronberg, G., Lindmark, G., Björk, S. (1988). Mass development of the flagellate Gonyostomum semen (Raphidophyta) in Swedish forest lakes - an effect of acidification? Hydrobiologia, 161, 217-236.

Druvietis, I. (2007). Gonyostomum semen (Ehrb.) Diesing expansion in Latvia's lakes. In: Abstracts of the $4^{\text {th }}$ International Conference "Research and Conservation of Biological Diversity in Baltic Region”. Daugavpils University, Daugavpils.

Farrell, C.A., Doyle, G.J. (2003). Rehabilitation of industrial cutaway Atlantic blanket bog in County Mayo, North-West Ireland. Wetlands Ecol. Manag., 11, 21-35.

Findlay, D., Paterson, J., Hendzel, L., Kling, H. (2005). Factors influencing Gonyostomum semen blooms in a small boreal reservoir lake. Hydrobiologia, 533(1), 243-252.

Girard, M., Lavoie, C., Theriault, M. (2002). The regeneration of a highly disturbed ecosystem: A mined peatland in Southern Quebec. Ecosystems, 5, 274-288.

Gorham, E., Rochefort, L. (2003). Peatland restoration: A brief assessment with special reference to Sphagnum bogs. Wetlands Ecol. Manag., 11, 109-119.

Haglund, A.L., Tornblom, E., Bostrom, B., Tranvik, L. (2002). Large differences in the fraction of active bacteria in plankton, sediments, and biofilm. Microb. Ecol., 43(2), 232-241.

Heino, J. (2000) Lentic macroinvertebrate assemblage structure along gradients in spatial heterogeneity, habitat size and water chemistry. Hydrobiologia, 418, 229-242.

Hertkorn, N., Claus, H., Schmitt-Kopplin, P., Perdue, E.M., Filip, Z. (2002). Utilization and transformation of aquatic humic substances by autochthonous microorganisms. Environ. Sci. Technol., 36(20), 4334-4345.

Higgins, T., Colleran E. (2004). A comparative assessment of water quality in four experimental lakes created on Irish cutaway peatland. In: Wise Use of Peatlands (pp. 372-378). Päivänen, J. (ed.). Proceedings of the $12^{\text {th }}$ International Peat Congress. Vol. 1.

Hongve, D., Løvstad, Ø., Bjørndalen, K. (1987). Gonyostomum semen — a new nuisance to bathers in Norwegian lakes. Verh. Internat. Ver. Limnol., 23, 430-434.

Hörnström, E. (2002). Phytoplankton in 63 limed lakes in comparison with the distribution in 500 untreated lakes with varying $\mathrm{pH}$. Hydrobiologia, 470, 115-126.

Kent, A.D., Jones, S.E., Yannarell, A.C., Graham, J.M., Lauster, G.H., Kratz, T.K., Triplett, E.W. (2004). Annual patterns in bacterioplankton community variability in a humic lake. Microb. Ecol., 48, 550-560.

Klavins, M., Rodinov, V., Druvietis, I. (2003). Aquatic chemistry and humic substances in bog lakes in Latvia. Boreal Env. Res., 8, 113-123.

Klavins, M., Rodinovs, V., Kokorite, I. (2002). Chemistry of Surface Waters in Latvia. Rīga: LU. 286 pp.

Krauklis, I. (1998). Sedas tīrelis [Seda Bog]. Grām.: Enciklopēdija. Latvijas daba (68. lpp.). Vol. 5. Rīga: Preses nams (in Latvian).
Lally, H., Higgins, T., Colleran, E., Gormally, M. (2008). Lakes: A new concept for wildlife conservation on Irish cutaway peatlands. In: After Wise Use - the Future of Peatlands. Vol. 1. (pp. 409-413). Farrell, C., Feehan, J. (eds.). Proceedings of the $13^{\text {th }}$ International Peat Congress. Tullamore.

Lamers, L.P.M., Smolders, A.J.P., Roelofs, J.G.M. (2002). The restoration of fens in the Netherlands. Hydrobiologia, 478, 107-130.

Larmanis, V. (2003) Evaluation of Threats to Biodiversity and Preparation of Conservation and Management Plan for Nature Reserve "Seda marsh" in North Vidzeme Biosphere Reserve. Final Report. Research study in the framework of the UNESCO Programme on Man and the Biosphere.

Lavoie, C., Grosvernier, P., Girard, M., Marcoux, K. (2003). Spontaneous revegetation of mined peatlands: An useful restoration tool? Wetlands Ecol. Manag., 11, 97-107.

Lepistö, L., Antikainen, S., Kivinen, J. (1994). The occurance of Gonyostomum semen (Ehr.). Diesing in Finnish lakes. Hydrobiologia, 273, 1-8.

Lindstrom, E.S. (2000). Bacterioplankton community composition in five lakes differing in trophic status and humic content. Microb. Ecol., 40(2), 104-113.

Lundin, L., Lode, E., Strömgren, M., Nilsson, T. (2008). Wetland: Wise after-use at terminated peat cuttings. In: After Wise Use - the Future of Peatlands. Vol. 1 (pp. 414-417). Farrell, C., Feehan, J. (eds.). Proceedings of the $13^{\text {th }}$ International Peat Congress. Tullamore.

McNally, G. (1998). Optimizing the return to Bord na Mona. In: The Future Use of Cutaway Bogs (pp. 13-16). Egan, T. (ed.). Proceedings of the first comprehensive conference on cutaway bogland rehabilitation. Offaly, Ireland.

Piper, A.M. (1944). A graphical procedure in the geochemical interpretation of water-analysis. Amer. Geophys. Union Trans., 25, 914-923.

Tadonleke, R.D., Planas, D., Lucotte, M. (2005). Microbial food webs in boreal humic lakes and reservoirs: Ciliates as a major factor related to the dynamics of the most active bacteria. Microb. Ecol., 49(2), 325-341.

Urtane, L., Klavins, M. (1995). Zooplankton communities of a group of lakes with different contents of humic substances in Latvia. Case study: State Teiču Bog Reserve. Proc. Latvian Acad. Sci., Sect. B, No. 1/2, 134-140.

Utermohl, H. (1958). Zur Vervollkommnung der quantitativen Phytoplankton-Methodik. Mitt. Internat. Verein. Limnol., 9, 1-38.

Van Duinen, G.A., Timm, T., Smolders, A.J.P., Brock, A.M.T., Verberk, W.C.E.P., Esselink, H. (2006). Differential response of aquatic oligochaete species to increased nutrient availability - a comprehensive study between Estonian and Dutch raised bogs. Hydrobiologia, 564, 143-155.

Vrede, K., Stensdotter, U., Lindstrom, E.S. (2003). Viral and bacterioplankton dynamics in two lakes with different humic contents. Microb. Ecol., 46(4), 406-15.

Ward, J.V. (1992) Aquatic Insect Ecology. 1. Biology and Habitat. Chichester: John Wiley \& Sons Inc. 438 pp.

Романенко В.И., Кузнецов С.И. (1974). Экология микроорганизмов пресных водоемов. Лабораторное руководство [Ecology of Freshwater Microorganisms. Guidance manual]. Ленинград: Наука (in Russian).

Received 12 March 2010

\section{ŪDEN̦U KVALITĀTE KŪDRAS KARJERU EZEROS SEDAS PURVĀ}

Pēdējos gados kā viena no iespējām izstrādāto kūdras karjeru rekultivācijai tiek piedāvāta seklu ezeru veidošana. Šì darba ietvaros tika pētīti applūdušie kūdras karjeri Sedas purvā ar mērķi novērtēt ūdens kvalitāti un noteikt galvenos faktorus, kas ietekmē ūdens kvalitāti applūdušajās teritorijās. Pētījumā konstatēts, ka Sedas purva teritorijās esošo applūdušo kūdras karjeru kīmiskais sastāvs (īpaši pēc galveno neorganisko jonu satura, $\mathrm{pH}$ un ūdens krāsainības), kā arī makrozoobentosa, fitoplanktona un mikroorganismu cenozes atškiras no tipisku purva ezeru ūdeṇu kvalitātes rādītājiem. Tas varētu liecināt par to, ka ūdeṇu sastāvu apsekotajās ūdenstilpēs ietekmē teritorijas ǵeoloğiskā uzbūve un gruntsūdeṇu ḳimmiskais sastāvs, jo pēc kūdras izsmelšanas karjeros nav atstāts pietiekams kūdras slānis. Turklāt Sedas purva applūdušajos karjeros pavasara palu laikā vērojama arī virszemes ūdeņu pieplūde no Sedas upes. Jau tuvākajā nākotnē būtu nepieciešams noteikt izstrādātā mitrāja atjaunošanas mērki konkrētās teritorijās — dabiska reǵenerācija ar minimālu cilvēka iejaukšanos vai arī mērḳtiecīgi virzīta darbība, lai veidotu bioloğiski daudzveidīgas un saimnieciski izmantojamas platības. 